\title{
Improving long-term survival by preventing early complications after lung transplantation: Can we prevent ripples by keeping pebbles out of the water?
}

\author{
Katie Kinaschuk, BSc, ${ }^{\mathrm{a}, \mathrm{b}}$ and Jayan Nagendran, MD, PhD, FRCSC ${ }^{\mathrm{a}, \mathrm{b}, \mathrm{c}, \mathrm{d}}$
}

\footnotetext{
From the ${ }^{\mathrm{a}}$ Department of Surgery, ${ }^{\mathrm{b}}$ Mazankowski Alberta Heart Institute, Alberta Health Services, and ${ }^{\mathrm{c}}$ Alberta Transplant Institute, University of Alberta, Edmonton, Alberta, Canada; and the ${ }^{\mathrm{d} C a n a d i a n}$ National Transplant Research Program, Edmonton, Alberta, Canada.

Disclosures: Authors have nothing to disclose with regard to commercial support.

Received for publication Dec 21, 2015; accepted for publication Dec 21, 2015; available ahead of print Jan 29, 2016.

Address for reprints: Jayan Nagendran, MD, PhD, FRCSC, Division of Cardiac Surgery, Department of Surgery, University of Alberta and Mazankowski Alberta Heart Institute, 2D2.18 WMC, 8440-112 St, Edmonton, Alberta T6G-2B7, Canada (E-mail: jayan@ualberta.ca).

J Thorac Cardiovasc Surg 2016;151:1181-2

$0022-5223 / \$ 36.00$

Copyright (C) 2016 by The American Association for Thoracic Surgery

http://dx.doi.org/10.1016/j.jtcvs.2015.12.045
}

Lung transplantation volumes continue to rise, with recipient waiting lists also growing, as a result of progression to end-stage lung disease by more patients and of improvements in lung transplant outcomes. ${ }^{1}$ Nonetheless, lung transplants continue to have the lowest long-term survival of all solid organ transplants. ${ }^{2,3}$ There is a need for ongoing quality measures and critical appraisal of shortand long-term outcomes to understand better where opportunities exist to improve long-term survival. Lung transplantation literature has been dichotomous, with studies describing either short- or long-term outcomes and lacking the relationship between long-term outcomes and the influence of early complications. Long-term survival is negatively associated with many perioperative and inhospital complications, including severity of primary graft dysfunction. ${ }^{4}$ There is, however, an overall paucity of clinical investigation attempting to stratify weight and specificity of the correlation of in-hospital complications with long-term survival.

In their article in this issue of the Journal, Chan and colleagues $^{5}$ have identified the weighted sum of risks for inhospital complications predicting their cumulative negative impact on long-term survival in a retrospective analysis of 748 patients undergoing lung transplants at the University of Pittsburgh Medical Center. Chan and colleagues ${ }^{5}$ used the Accordion Severity Grading System (ASGS) to classify adverse events as weighted by quantifying total morbidity burden. The study reveals that more than $90 \%$ of patients have a complication after the lung transplant, and the presence of any complication is negatively correlated with longterm survival. Those with the greatest negative impact included renal, cardiac, hepatic, and vascular complications. The ASGS sum was also predictive of decreased long-term survival in a multivariate analysis, alluding to the ripple effect of early complications post-lung dures. ${ }^{6-9}$

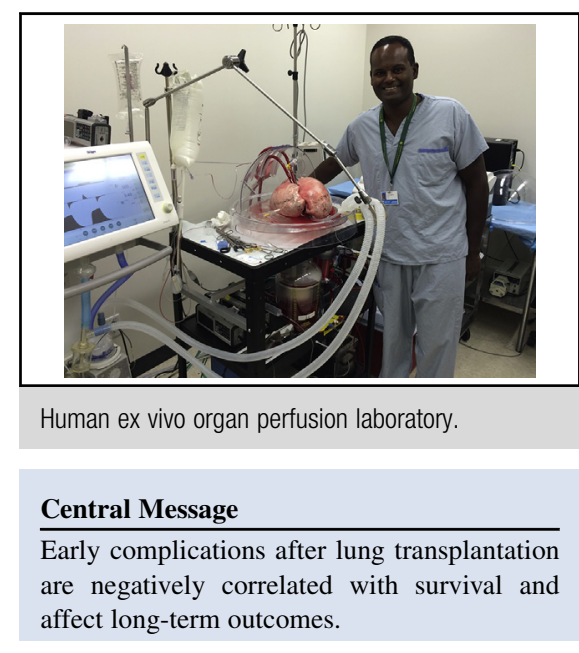

See Article page 1171 .

transplantation having major long-term implications with regard to decreased survival. Given that complications are nearly ubiquitous (93\% of patients) at one of the leading thoracic transplantation centers, the significance of these results highlights the need for better management strategies for postoperative complications. The early identification through lung transplant-specific monitoring and intervention protocols may indeed represent critical opportunities to improve outcomes at all thoracic transplant programs, as has proved to be successful for other surgical proce-

The ASGS was validated in the study of Chan and colleagues ${ }^{5}$ for its predictive value in negatively correlating long-term survival with the weighted ASGS sum after lung transplants. This scoring system is strengthened by the lessened variance in complication recognition and treatment strategies within the single center of this study. Chan and colleagues ${ }^{5}$ do, however, appreciate that institutional variances may occur with identification and treatment algorithms of complications, such that it would require institution-specific implementation and validation of the ASGS system before using the weighted sum ASGS to influence their practice.

The evolution of lung transplantation has been rapid, with many incremental improvements in preservation, surgical technique, and immunosuppression contributing to improved early and late allograft function. The article by 
Chan and colleagues ${ }^{5}$ from the University of Pittsburgh Medical Center interestingly shows a significantly stronger correlation by multivariate analysis between nonpulmonary complications and decreased long-term survival. Because the incidence of any complication is negatively associated with long-term survival, however, it is important to appreciate that the complications of highest incidence still include pulmonary $(72 \%)$ and infectious $(70 \%)$ complications. Thus despite the need to improve treatment algorithms of highly predictive non-allograft related complications, the greatest opportunity to decrease the overall rates of complications still exists within pulmonary and infectious etiologies.

Results from lung transplant clinical trials often focus on the severity of primary graft dysfunction and inhospital survival as primary end points. The study of Chan and colleagues ${ }^{5}$ provides a rationale for the inclusion of significant morbidity caused by major non-allograft related postoperative complications (renal, cardiac, hepatic, and vascular) to validate efficacy further with long-term harmful implications in clinical trials. Although this study was not designed to investigate the correlation of pretransplant factors with postoperative events, it is noteworthy that Charlson Comorbidity Index values ${ }^{10}$ were not found to be a predictor of long-term survival $(P=.67)$, suggesting that operative and donor factors may be even more predicative of postoperative complications linked to decreased long-term survival. This may represent the need for careful reporting and consideration of non-allograft related postoperative complications in assessing new technologies for donor lung management, including clinical trials examining the efficacy of ex vivo lung perfusion. ${ }^{11-13}$

The appreciation of the ripple effect by which early postoperative complications negatively affect long-term survival provides physicians with an awareness to improve treatment strategies of specific complications. It may warrant more vigilant long-term surveillance once a complication has occurred. Ultimately, determination of preventive measures by identifying predictors of complications will have the greatest positive effect on survival, and this area still needs further investigation to decrease the overall rates early complications.

\section{References}

1. Yusen RD, Edwards LB, Kucheryavaya AY, Benden C, Dipchand AI, Goldfarb SB, et al. The registry of the International Society for Heart and Lung Transplantation: thirty-second official adult lung and heart-lung transplantation report-2015; focus theme: early graft failure. J Heart Lung Transplant. 2015;34:1264-77.

2. Yusen RD, Shearon TH, Qian Y, Kotloff R, Barr ML, Sweet S, et al. Lung transplantation in the United States, 1999-2008. Am J Transplant. 2010;10(4 Pt 2): 1047-68.

3. Valapour M, Paulson K, Smith JM, Hertz MI, Skeans MA, Heubner BM, et al. OPTN/SRTR 2011 annual data report: lung. Am J Transplant. 2013;13(Suppl 1):149-77.

4. Lee JC, Christie JD. Primary graft dysfunction. Clin Chest Med. 2011;32: 279-93.

5. Chan EG, Bianco V III, Richards T, Hayanga JW, Morrell M, Shigemura N, et al. The ripple effect of a complication in lung transplantation: evidence for increased long-term survival risk. J Thorac Cardiovasc Surg. 2016;151: 1171-80.

6. Ghaferi AA, Birkmeyer JD, Dimick JB. Complications, failure to rescue, and mortality with major inpatient surgery in Medicare patients. Ann Surg. 2009; 250:1029-34.

7. Livingston EH, Cao J. Procedure volume as a predictor of surgical outcomes. JAMA. 2010;304:95-7.

8. Ghaferi AA, Birkmeyer JD, Dimick JB. Variation in hospital mortality associated with inpatient surgery. $N$ Engl J Med. 2009;361:1368-75.

9. Kilic A, George TJ, Beaty CA, Merlo CA, Conte JV, Shah AS. The effect of center volume on the incidence of postoperative complications and their impact on survival after lung transplantation. J Thorac Cardiovasc Surg. 2012;144:1502-8; discussion 1508-9.

10. Charlson ME, Pompei P, Ales KL, MacKenzie CR. A new method of classifying prognostic comorbidity in longitudinal studies: development and validation. $J$ Chronic Dis. 1987;40:373-83.

11. Cypel M, Yeung JC, Liu M, Anraku M, Chen F, Karolak W, et al. Normothermic ex vivo lung perfusion in clinical lung transplantation. N Engl J Med. 2011;364: 1431-40.

12. Warnecke G, Moradiellos J, Tudorache I, Kühn C, Avsar M, Wiegmann B, et al. Normothermic perfusion of donor lungs for preservation and assessment with the Organ Care System Lung before bilateral transplantation: a pilot study of 12 patients. Lancet. 2012:380:1851-8.

13. Sanchez PG, Rajagopal K, Pham SM, Griffith BP. Defining quality during ex vivo lung perfusion: The University of Maryland experience. J Thorac Cardiovasc Surg. 2015;150:1376-7. 